Anna Ciostek

Uniwersytet Warszawski

\title{
TERMINOLOGIE DE L'UNION EUROPÉENNE EN POLONAIS : ENTRE IDENTITÉ DU TRADUCTEUR ET CELLE DU DESTINATAIRE
}

\author{
The EU law terminology: \\ between the identity of translator and that of recipient
}

\begin{abstract}
The EU law terminology has enriched Polish legal language since Poland's accession to the EU and has been subject to changes. Apart from the terms borrowed from English and French, there are more and more polonized equivalents, closer to the linguistic identity of their recipients. At the same time, in the general language, we observe the creation of equivalents of these terms in other registers. They are calles bruxelisms, which are evolving and being terminologized.
\end{abstract}

Keywords: bruxelism, European Union, evolution, identity, terminology

La terminologia del diritto dell'Unione Europea: tra l'identità del traduttore e quella del destinatario

Riassunto

La terminologia degli atti normativi emanati dall'Unione Europea arricchisce la lingua della legge polacca a partire dall'entrata della Polonia nell'Unione Europea ed è soggetta ai continui cambiamenti. Oltre ai termini presi in prestito dall'inglese o dal francese, ci sono sempre più corrispondenti polonizzati che si identificano più vicini all'identità linguistica del territorio. Simultaneamente viene osservato, all'interno della lingua standard, un fenomeno di creazione dei termini equivalenti in altri registri ovvero dei derivati comunitari (denominati dall'autrice stessa «bruxellismi») che sono in via di evoluzione e terminologizzazione.

Parole chiave: derivati comunitari, Unione Europea, evoluzione, identità, terminologia

\section{Contexte}

La terminologie propre aux actes officiels publiés par l'Union européenne se forge depuis une soixantaine d'années. Incorporée massivement en polonais comme langue réceptrice il y a 15 ans, avec la traduction de l'acquis communautaire, elle reste 
nouvelle. Cette terminologie est le fruit de débats et de compromis entre spécialistes des services linguistiques des institutions européennes issus de différentes cultures et traditions juridiques et linguistiques. Les textes juridiques où elle est employée revêtent un caractère hybride dû au contact et aux influences réciproques de quelques dizaines de langues (Doczekalska) lors du processus de création du droit del'Union européenne. En même temps, les langues juridiques nationales se voient déculturer. Déculturer signifie ici perdre (une partie) de son identité culturelle et/ ou linguistique au profit de concepts et termes nouveaux, qui 1. relèvent du droit européen autonome et 2 . répondent aux besoins du multilinguisme officiel prôné parl'Union européenne, processus impliquant la traduction.

\section{Qu'est-ce que l'identité}

L’identité est un terme polysémique qui renvoie entre autres à la similitude, à la communauté, à l'unité (Petit Robert), et dont les significations se sont développées à travers différents domaines : philosophie (depuis les philosophes présocratiques jusqu'à Hegel), psychologie (Freud), sciences sociales (Erikson), mathématiques, logique, géographie, etc. L'identité peut se décliner en identité individuelle, personnelle (concepts de mêmeté et d'ipséité chez Ricœur) ou identités collective et institutionnelle.

\subsection{L'identité au sein de l'Union européenne}

Dans le contexte européen qui nous intéresse, par identité, nous entendons identité linguistique et identité culturelle comme notions qui découlent du sentiment d'appartenance à une langue et à une culture, ainsi que la reconnaissance des traits caractéristiques y afférents par un individu ou par les autres. Il s'agit, en l'occurrence, de plusieurs types d'identité : celle de l'émetteur (UE) qui crée le message (concept et terme européen) et celle du récepteur (citoyen européen) qui le reçoit par le canal de la traduction.

En dehors des acceptions précitées que prend le concept d'identité selon les différents domaines et points de vue, il faut envisager la perspective adoptée par l'Union européenne qui, elle aussi, fait de l'identité un concept central de ses réflexions, incarné par l'idée d'identité européenne commune aux 27 Etats membres avec leurs 24 langues.

En dépit d'un riche héritage commun que partagent les peuples d'Europe et un vaste programme d'actions culturelles et éducatives déployé par l'Union européenne pour, d'abord, favoriser l'émergence d'une identité européenne et renforcer le sentiment de ladite identité par la suite, cette dernière ne s'est pas construite à ce jour. Chaque Etat membre apporte à l'Union sa ou ses langue.s, son histoire, sa culture, ses us et coutumes sans être prêt à y renoncer. Un 
sondage réalisé en avril 2019 par le Centre polonais de recherche sur l'opinion publique (CBOS, 2019) montre que les Polonais s'identifient de plus en plus à l'Union européenne. Cependant, 15 ans après l'adhésion de la Pologne à l'UE, ils sont 50\% environ à se dire Européens (au sens de l'UE), contre presque $100 \%$ à se définir comme Polonais, l'identité européenne restant secondaire par rapport à l'identité nationale, même si $72 \%$ des Polonais interrogés dans le cadre d'Eurobaromètre (2015) se sentent attachés à la citoyenneté européenne. Il est d'autant plus difficile de s'identifier à l'Union qu'elle ne cesse de s'interroger sur son identité, dans une réalité en constant mouvement où les concepts foisonnent : organisation internationale, fédération, Union à plusieurs vitesses, Europe à la carte... Pour cette raison, « Le discours sur l'identité européenne sonnera creux tant qu'il ne parviendra pas à s'articuler autour d'un contenu identifiable $\gg$ (Wismann, 2014).

\subsection{L'identité linguistique dans l'Union européenne}

L'intégration européenne qui se fait aujourd'hui, passe par différentes étapes dont chacune laisse son empreinte dans la langue, puissant outil de construction identitaire et communautaire. Mais quelle langue? Parmi les 24 langues officielles de l'UE seules 3 sont véhiculaires (anglais, français, allemand), avec une prédominance toujours croissante de l'anglais dont la part en tant que langue de l'original a atteint $82 \%$, en 2016, selon les données de la Commission européenne ${ }^{1}$, contre $4 \%$ pour le français et $2 \%$ pour l'allemand, les autres 21 langues ne représentant au total que $12 \%$. Or, comme le constate Claude Hagège (2003) :

le contenu symbolique des langues correspond à leur pouvoir identitaire, c'està-dire au reflet qu'elles offrent des nations les plus variées. L'attachement de ces dernières à leurs langues est le plus souvent très puissant et peut être générateur de conflit.

Guidée par la devise «Unie dans la diversité », l’UE cherche à concilier différentes traditions linguistiques et juridiques pour construire sa terminologie et trouver des compromis assurant la transparence et la sécurité juridique des termes. A la création d'un terme au sein des institutions européennes travaillent des linguistes, juristes, linguistes-juristes, terminologues et traducteurs représentant des cultures, systèmes juridiques, traditions et pays différents et communiquant pendant un débat qui doit aboutir à l'élaboration du terme dans une langue qui n'est pas forcément la leur. Cet amalgame d'expériences,

${ }^{1}$ Komisja Europejska : Jezzyk oryginałów, https://ec.europa.eu/commission/ index_pl (consulté le 30.06.2019). 
de visions et de sensibilités individuelles aboutit souvent à la naissance d'internationalismes propres à l'UE (directive, intervention, dialogue, exclusion numérique, harmonisation...), ce qui contribue à forger une sorte d'identité terminologique de l'Union.

\subsection{L'identité terminologique de l’Union européenne}

Chercher son identité c'est aussi se différencier. L'Union européenne a instauré un ordre juridique autonome, porteur de concepts uniques, doté d'une terminologie spécifique sans équivalents dans le droit national des Etats membres ou le droit international, employée dans un ensemble de textes relevant du droit primaire/originaire (composé par les traités) et du droit dérivé (règlements, directives, décisions), rédigés, et plus exactement traduits, dans les 24 langues officielles de l'Union. Qui dit identité, dit aussi identique: toutes les versions traduites passent pour identiques, ou, du moins, elles ont une valeur juridique identique. Quasi identiques sont également certaines eurolexies, dont beaucoup sont de nouvelles notions supranationales: uniques quant au contenu et quant à la forme. Leur isomorphisme est bien visible à l'exemple du terme subsidiarité, adopté et adapté par 20 des 24 langues de l'UE (d'après IATE) :

1. pl subsydiarność / zasada pomocniczości

2. bg принцип на субсидиарност

3. cs zásada subsidiarity

4. da nærhedsprincip

5. de Subsidiaritätsprinzip

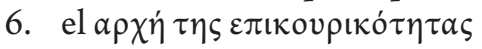

7. en principle of subsidiarity

8. es principio de subsidiariedad

9. et subsidiaarsuse põhimõte

10. fi toissijaisuusperiaate

11. fr principe de subsidiarité

12. ga prionsabal na coimhdeachta

13. hr načelo supsidijarnosti

14. hu a szubszidiaritás elve

15. it principio di sussidiarietà

16. It subsidiarumo principas

17. lv subsidiaritātes princips

18. $\mathrm{mt}$ princiipju tas-sussidjarjetà

19. $\mathrm{nl}$ subsidiariteitsbeginsel

20. pt princípio da subsidiariedade

21. ro principiul subsidiarității

22. sk zásada subsidiarity

23. sl načelo subsidiarnosti

24. sv subsidiaritetsprincipen 
Il est intéressant de se demander cependant si les notions supranationales ainsi forgées sont aussi supraidentitaires. Selon Roger Goffin (1994 : 640) les langues et traditions juridiques ont quand-même laissé leur empreinte sur le droit communautaire: p. ex. le droit de la concurrence s'inspirerait $\mathrm{du}$ droit allemand en la matière, tandis que celui sur le statut de la fonction publique européenne serait décalqué du texte français. Traduits, les « textescompromis (...) laissent transparaître en filigrane les modes d'expression du modèle $\gg$.

Ainsi, les grandes langues véhiculaires, imprègnent-elles les autres langues nationales sur le plan lexical et sémantique, la traduction se faisant à partir de la langue dominante, même si les institutions européennes, de par leur choix officiel de ne favoriser aucune langue, prônent le multilinguisme institutionnel et l'égalité des 24 langues.

\section{Terminologie européenne traduite en polonais vs bruxellismes}

Le polonais accueille donc des termes appartenant à l'eurolecte, issus d'autres langues et cultures, uniques et souvent unifiés ou traduits littéralement. Ainsi, les néologismes européens en polonais s'inspirent-ils des précédents dans d'autres langues et résultent d'un processus que Hermans et Vansteelandt (1999) appellent néologie traductive.

Au lieu d'être transparentes, beaucoup de ces lexies restent ésotériques pour le non-spécialiste: des mots empruntés au lexique commun se voient utiliser dans un nouveau sens spécialisé, ayant certes une portée et des implications juridiques, mais qui paraissent étranges et étrangers. Des néosémantismes comme kwota (quota), mapa drogowa (feuille de route), semestr europejski (semestre européen), zielona księga (livre vert) acquièrent ainsi une nouvelle identité, sans être désambiguïsés. D’autres néologismes, notamment : kwalifikowalność wydatków (éligibilité des dépenses), komitologia (comitologie), zrównoważoność (durabilité) ou identyfikowalność (traçabilité) sont loin d'être lexicalisés.

Certes, la terminologie européenne fait partie d'une langue de spécialité parmi d'autres et doit comporter des termes conçus par et pour les spécialistes, mais contrairement à la plupart des langues de spécialité, elle n'est pas destinée qu'aux professionnels. A savoir, le droit de l’Union a un effet direct dans les Etats membres et touche les citoyens européens dans leur quotidien socioprofessionnel. Les actes émis par l'UE, tels que directives, recommandations ou avis ayant force obligatoire sont susceptibles de s'adresser directement à des particuliers, comme les décisions par exemple. Le règlement, 
un acte de portée générale, obligatoire dans tous ses éléments, est aussi directement applicable dans chaque Etat membre ${ }^{2}$.

Il paraît donc naturel de veiller à la lisibilité de ces notions, eu égard à l'implication des citoyens européens. Cependant, de nombreux termes issus du droit communautaire revêtent un caractère opaque pour le profane qui est destinataire de ces différents actes traduits dans les langues nationales. L'identité linguistique du traducteur, à qui incombe aussi le rôle du dernier maillon du processus législatif ${ }^{3}$, se heurte à celle du destinataire. Pour s'approprier les concepts véhiculés par la terminologie officielle, le destinataire polonais crée des équivalents dans la langue générale, plus proche de son vécu et de ses traditions. Nous les avons appelés : bruxellismes (Ciostek, 2013) ${ }^{4}$.

\subsection{Bruxellismes comme équivalents de noms propres}

Les unités linguistiques qui font partie des bruxellismes appartiennent au registre non-standard de la langue que l'on pourrait classer de différentes manières : comme argot (« un phénomène lexical qui consiste à créer des termes qui doublent le vocabulaire usuel $\gg)^{5}$, jargon (parler technique) ou jargot (Sourdot, 1991) en fonction de leur degré de spécialisation et de lisibilité. Une pratique fréquemment rencontrée en polonais consiste à remplacer les noms propres d'institutions européennes, de documents ou de fonctions, par un équivalent proche de l'identité culturelle du destinataire.

\subsubsection{Bruxellismes remplaçant les noms de traités européens}

Dans les documents officiels de l'UE sont utilisées, à côté des noms de traités complets, également leurs formes abrégées. A titre d'exemple, Le traité sur l'Union européenne se réduit à l'appellation Traité de Maastricht ou à l'acronyme TUE. Dans la langue générale, ce procédé va plus loin, pour aboutir à la réduction du nom de traité à la seule ville où il a été signé : Maastricht, tout en copiant les structures qui sont connues (traité de Versailles vs Versailles) ${ }^{6}$, etc.

${ }^{2}$ http://ec.europa.eu/civiljustice/glossary/glossary_fr.htm (consulté le 30.06.2019).

${ }^{3} \mathrm{http}: / /$ www.europarl.europa.eu/about-parliament/fr/organisation-and-rules/ multilingualism (consulté le 30.06.2019).

${ }^{4}$ Intervention à l'Institut d'Etudes romanes en mars 2013.

${ }_{5}^{5}$ Définition de Denise François-Geiger citée par Marc Sourdot (1991).

${ }^{6}$ Cf. le titre du livre de Lujo Brentano Ce que l'Allemagne a payé depuis Versailles: Les prestations de l'Allemagne en vertu du Traité, https://www.goodreads.com/book/ show/17098722-ce-que-l-allemagne-a-pay-depuis-versailles?rating=2 (consulté le 30.06.2019). 
Tableau 1. Bruxellismes remplaçant les noms de traités européens

\begin{tabular}{|l|l|l|}
\hline Nom officiel du traité (titre intégral) & Nom abrégé & Bruxellisme \\
\hline $\begin{array}{l}\text { Traktat o Unii Europejskiej } \\
\text { Le traité sur l'Union européenne (TUE) }\end{array}$ & Traktat z Maastricht & Maastricht \\
\hline $\begin{array}{l}\text { Traktat Amsterdamski zmieniający } \\
\text { Traktat o Unii Europejskiej. Traktaty }\end{array}$ & $\begin{array}{l}\text { Traktat amsterdamski } \\
\text { Traktat z Amsterdamu }\end{array}$ & Amsterdam \\
ustanawiające Wspólnoty Europejskie \\
$\begin{array}{l}\text { i niektóre związane z nimi akty } \\
\text { Traité d'Amsterdam modifiant le traité sur } \\
\text { l’Union européenne, les traités instituant } \\
\text { les Communautés européennes et certains } \\
\text { actes connexes }\end{array}$ & & \\
\hline $\begin{array}{l}\text { Traktat o Funkcjonowaniu Unii } \\
\text { Europejskiej } \\
\text { Traité sur le fonctionnement de l’Union } \\
\text { européenne }\end{array}$ & Traktatlizboński & Lizbona \\
\hline
\end{tabular}

Source : Élaboration propre.

Les bruxellismes sont ensuite véhiculés par la presse, p.ex. : « Czeski TK powiedzial 'tak' dla Lizbony $\gg^{7}$, « Pióro, które nie chciało podpisać 'Lizbony' - na licytację $»^{8}$, « WPZiB i EPBiO od Maastricht do Lizbony » ${ }^{9}$, « Unia podjęla próbę reformy traktatowej pięciokrotnie (począwszy od traktatu z Maastricht, poprzez Amsterdam, Niceę, traktat konstytucyjny i wreszcie lizboński) $\gg^{10}$.

\subsubsection{Bruxellismes remplaçant les noms d'institutions ou de fonctions}

Le destinataire polonais use de son imagination pour remplacer les noms d'institutions européennes et les appeler de manière succincte. Ainsi, le président du Conseil européen est dénommé président de l'Europe, chef $d u R E$, ou encore, au registre familier, roi de l'Europe. La fonction de député au Parlement

7 https://www.polskieradio.pl/5/3/Artykul/189732,Czeski-TK-powiedzial-takdla-Lizbony (consulté le 29.11.2018).

8 https://fakty.interia.pl/polska/news-pioro-ktore-nie-chcialo-podpisaclizbony-na-licytacje,nId,869173\#utm_source=paste\&utm_medium=paste\&utm campaign=firefox (consulté le 30.06.2019).

9 http://webcache.googleusercontent.com/search?q=cache:u4EA3wH7XncJ: uniaeuropejska.org/analiza-uniaeuropejska-org-wpzib-i-epbio-od-maastricht-do-liz bony/+\&cd=27\&hl=pl\&ct=clnk\&gl=pl\&client=firefox-b-d (consulté le 30.06.2019).

10 http://krytykapolityczna.pl/swiat/ue/te-wybory-mialy-zmienic-unieskonczylo-sie-jak-zawsze/ (consulté le 29.11.2018). 
européen prend trois synonymes en polonais courant, se résumant au sens de 'europarlementaire' ou 'eurodéputé'.

Tableau 2. Bruxellismes remplaçant les noms d'institutions et de fonctions

\begin{tabular}{|l|l|}
\hline \multicolumn{1}{|c|}{ Terme officiel } & \multicolumn{1}{c|}{ Bruxellisme } \\
\hline $\begin{array}{l}\text { Parlament Europejski } \\
\text { Parlement européen }\end{array}$ & $\begin{array}{l}\text { Europarlament } \\
\text { Unijny Parlament }\end{array}$ \\
\hline $\begin{array}{l}\text { Poseł do Parlamentu Europejskiego } \\
\text { Député au Parlemenr européen }\end{array}$ & $\begin{array}{l}\text { członek PE } \\
\text { europarlamentarzysta } \\
\text { eurodeputowany } \\
\text { europoset }\end{array}$ \\
\hline Przewodniczący Rady Europejskiej & $\begin{array}{l}\text { prezydent Europy } \\
\text { pról Europy } \\
\text { Président du Conseil européen }\end{array}$ \\
& $\begin{array}{l}\text { szefady Europejskiej } \\
\text { szef RE }\end{array}$ \\
\hline
\end{tabular}

Source : Élaboration propre.

Dans la presse, le nom Parlement européen suit le même modèle : « Oto nowy europarlament. Kto nam urządzi życie w Unii ? $\gg^{11}$.

\subsubsection{Bruxellismes remplaçant les noms de groupes de pays}

Certains bruxellismes servent à remplacer les noms de groupes de pays réunis autour d'un concept commun, comme par exemple les pays de la zone euro, c'està-dire les États membres de l'Union européenne qui ont adopté l'euro. Pour ce terme officiel, le polonais courant a trouvé au moins six synonymes :

Tableau 3. Bruxellismes remplaçant les noms de groupes de pays

\begin{tabular}{|l|l|}
\hline \multicolumn{1}{|c|}{ Terme officiel } & \multicolumn{1}{|c|}{ Equivalent en polonais courant } \\
\hline Strefa euro/obszar euro & Eurogrupa \\
Zone euro & Euroland \\
& Euroobszar \\
& Eurostrefa \\
& Eurozona \\
& strefa Eurolandu \\
\hline
\end{tabular}

Source : Élaboration propre.

${ }^{11} \mathrm{http}$ //biqdata.wyborcza.pl/biqdata/7,159116,24848414,oto-nowy-europarlamentkto-nam-urzadzi-zycie-w-unii-infografiki.html (consulté le 29.11.2018). 
Une tendance similaire concerne le terme les pays du groupe de Visegrád, transformé en nombreux bruxellismes suivants :

Tableau 4. Bruxellismes remplaçant le terme Grupa Wyszehradzka

\begin{tabular}{|l|l|}
\hline \multicolumn{1}{|c|}{ Terme officiel } & \multicolumn{1}{c|}{ Equivalent en polonais courant } \\
\hline $\begin{array}{l}\text { Grupa Wyszehradzka } \\
\text { Groupe de Visegrád }\end{array}$ & V4 \\
& grupa V4 \\
& państwa grupy V4 \\
& kraje wyszehradzkie \\
& kraje V4 \\
& Wyszehrad \\
\hline
\end{tabular}

Source : Élaboration propre.

L’emploi de ces différents bruxellismes dans le discours public en Pologne devient de plus en plus fréquent. Médiatisé, cet équivalent courant est sur la voie de se terminologiser, son emploi sur le site officiel du Groupe en étant la preuve :

Grupa V4 nie powstała jako alternatywa dla ogólnej integracji ogólnoeuropejskiej, ani nie próbuje konkurować z istniejącymi już i funkcjonującymi strukturami środkowoeuropejskimi ${ }^{12}$.

\subsection{Terminologisation de bruxellismes}

A force d'être employés dans le discours public, notamment dans les médias, certains bruxellismes se glissent dans les actes officiels pour se terminologiser finalement.

Tel est l'exemple des titres de directives européennes (Ciostek, 2015), d'habitude longs et complexes, pour lesquels le destinataire trouve des formes lapidaires, exprimées en polonais par un adjectif ${ }^{13}$ :

12 http://www.visegradgroup.eu/v4-110412-1 (consulté le 30.06.2019).

13 En français, la même fonction est assurée par un nom en asyndète. 
Tableau 5. Terminologisation de bruxellismes

\begin{tabular}{|l|l|}
\hline \multicolumn{1}{|c|}{ Titre officiel } & $\begin{array}{c}\text { Équivalent du titre officiel } \\
\text { (bruxellisme) }\end{array}$ \\
\hline $\begin{array}{l}\text { Dyrektywa Parlamentu Europejskiego i Rady 2014/40/ } \\
\text { UE z dnia 3 kwietnia 2014 r. w sprawie zbliżenia przepisów } \\
\text { ustawowych, wykonawczych i administracyjnych państw } \\
\text { członkowskich w sprawie produkcji, prezentowania } \\
\text { i sprzedaży wyrobów tytoniowych i powiązanych wyrobów } \\
\text { oraz uchylająca dyrektywę 2001/37/WE }\end{array}$ & \\
Directive 2014/40/UE du Parlement européen et du Conseil \\
du 3 avril 2014 relative au rapprochement des dispositions \\
législatives, réglementaires et administratives des États \\
membres en matière de fabrication, de présentation et de vente \\
des produits du tabac et des produits connexes, et abrogeant la \\
directive 2001/37/CE
\end{tabular}

Source : Élaboration propre.

Ces formes raccourcies sont ensuite citées dans le contenu d'autres directives, p.ex. « identyfikacja i mapowanie szczególnych typów siedlisk, zwłaszcza uznanych lub określonych na mocy przepisów wspólnotowych (dyrektywa siedliskowa i dyrektywa ptasia) $\gg^{14}$.

Ainsi, certaines unités, après un stade non officiel de bruxellisme, deviennent termes, des termes qui répondent mieux à l'identité linguistique de leur utilisateur final.

14 Directive 2008/56/CE du Parlement Européen et du Conseil. 


\section{Conclusion}

Les bruxellismes ne sont pas uniquement affaire du polonais ou du destinataire polonais. Les langues d'autres Etats membres se sont adaptées également sur le plan du langage commun à ce qui semble plus proche de leur identité linguistique et culturelle. Ainsi, en français, sont nés p.ex. directive machines, eurodéputé (mais pas *Europarlement), eurozone, Visegrád 4, ou chef de la diplomatie européenne/ministre des Affaires étrangères de l'Union européenne (pour remplacer la charge de haut représentant de l'Union pour les affaires étrangères et la politique de sécurité). Le polonais qui a rejoint les langues officielles des Quinze il y a 17 ans à peine puise dans les termes préexistants, forgés dans les langues de ce qu'on appelle vieille Europe - 'pays fondateurs' (pol. stara Unia) et dans leurs variantes non standard.

Les exemples précités montrent que les destinataires de la législation européenne cherchent à remplacer, dans leurs langues respectives, les termes supranationaux inclus dans les actes officiels par des équivalents qui soient plus proches de leurs identités linguistique et culturelle, et donc plus facilement identifiables, ceci malgré un fort sentiment d'appartenance à l'Union européenne exprimé dans les sondages.

En même temps, l'identité du créateur des termes européens, celle du traducteur qui les fait véhiculer auprès du destinataire national se voient estomper au profit d'une nouvelle identité qui fait surface, à savoir celle des termes propres aux documents publiés par l'UE qui sont des notions uniques.

\section{Bibliographie}

Application Le Petit Robert pour iPad (consulté le 13.06.2019).

CBOS (2019) : 15 lat członkostwa Polski w Unii Europejskiej, https://cbos.pl/PL/ publikacje/news/2019/14/newsletter.php (consulté le 30.06.2019).

Ciostek, A. (2015) : Titres officiels français et polonais des directives européennes et ce qu'on en fait dans l'emploi courant. In : Sujecka-Zając, J., Pachocińska, E., Wróblewska-Pawlak, K. (éd.), Regards sur l'oral et l'écrit, pp. 25-33. Warszawa : Wydawnictwa Uniwersytetu Warszawskiego.

Doczekalska, A. : Język wielojęzycznego prawa Unii Europejskiej - (de)kulturyzacja $i$ (de)hybrydyzacja jezyka prawnego podczas tworzenia i transpozycji prawa Unii Europejskiej, https://projekty.ncn.gov.pl/index.php?projekt_id=241555 (consulté le 09.09.2021).

Eurobaromètre Standard 84 : La citoyenneté européenne, https://www.google.com/ $\mathrm{url} ? \mathrm{sa}=\mathrm{t} \& \mathrm{rct}=\mathrm{j} \& \mathrm{q}=\& \mathrm{esrc}=\mathrm{s} \&$ source $=$ web $\& \mathrm{~cd}=2 \& \mathrm{ved}=2 \mathrm{ahUKEwi} \mathrm{iZ}$ x2aDjAhXGb1AKHW_pCXsQFjABegQIAhAC\&url=https\%3A\%2F\%2Fec. europa.eu\%2Fcommfrontoffice\%2Fpublicopinion\%2Findex.cfm\%2FResultDoc\%2 
Fdownload\%2FDocumentKy\%2F72666\&usg=AOvVaw1GLvZuY85T20h5 olsFor1s (consulté le 30.06.2019).

Goffin, R. (1994) : L'eurolecte : oui, jargon communautaire : non. Meta, 39(4), pp. 636642, https://doi.org/10.7202/002930ar (consulté le 30.06.2019).

Hagège, C. (2003) : Théorie linguistique, http://claude.hagege.free.fr/ (consulté le 09.09.2014).

Hermans, A., Vansteelandt, A. (1999) : Neologie traductive. Terminologies Nouvelles (Nouveaux outils pour la neologie), 20, pp. 37-43.

IATE - Interactive Terminology for Europe, https://iate.europa.eu/home (consulté le 30.06.2019).

Komisja Europejska : Język oryginatów, https://ec.europa.eu/commission/index_pl (consulté le 30.06.2019).

Sourdot, M. (1991) : Argot, jargon, jargot! Langue française, 90, pp. 13-27.

Wismann, H. (2014) : Qu'est-ce que l'identité européenne ?, https://www.lemonde. $\mathrm{fr} /$ societe/article/2014/05/13/qu-y-a-t-il-d-europeen-dans-les-realiteseuropeennes_4415278_3224.html (consulté le 30.06.2019). 